\title{
SY64-2 Symposium
}

\section{Introduction of Treatment Concept of "anti-fibrosis" instead of "anti- inflammation" in interstitial lung diseases}

Arata Azuma

Department of Pulmonary Medicine and Oncology, Graduate School of Medicine, Nippon Medical School, Tokyo, Japan

Fibrosis is pathologically defined in all organs as the terminal mode of the organ. Today, with the introduction of HRCT examination, the UIP pattern is also widely used in routine practice as a form of the poorest prognosis in images. Moreover, while image inspection is inferior to judgment of pathological level, it carries superiority which can reproduce the change over time. In this field, however, the evaluation of the prognostic index based on remarkable development of molecular genetics and biomarkers was delayed.

On the other hand, in IPF, the change amount of respiratory function (FVC has the most reproducibility) is the most accurate prognostic index. Many clinical trials have shown scientific evidence in recent years.

Antifibrotic drugs, pirfenidone, resulted in Japan developing its own clinical trial, and the \% VC drops by about $50 \%$ annually. Prior to the European countries and the United States, it was approved as the IPF treatment drug only in Japan in 2008. Currently permits and licenses are spreading all over the world.

On the other hand, from the results of PANCER trial, treatment of corticosteroid for the suppression of inflammation has been proved to deteriorate the life prognosis of IPF, and it is recommended not to use it in GL. Therefore, instead of "anti-inflammation", "inhibition of fibrosis" has been shown to inhibit the progression of IPF and provably improve the prognosis of life. The second Nintedanib is three growth factor inhibitors developed for the same fibrosis inhibition purpose. It was distributed to the market from 2014.

ILD other than IPF, even if inflammation destroys the existing structure of the lung, fibrosis progresses with aging. There is a limit with anti-inflammatory medicine alone.

Due to the transition of treatment concept, the disease concept also changes. FVC decline is due to the progress of fibrosis. Biomarker research corresponding to disease behavior is underway. 